\title{
Rhombic alternative tableaux, assemblées of permutations, and the ASEP
}

\author{
Olya Mandelshtam $\|^{1}$ and Xavier Viennot ${ }^{2}$ \\ ${ }^{1}$ University of California, Berkeley \\ ${ }^{2}$ LaBRI, Bordeaux, France
}

\begin{abstract}
In this paper, we introduce the rhombic alternative tableaux, whose weight generating functions provide combinatorial formulae to compute the steady state probabilities of the two-species ASEP. In the ASEP, there are two species of particles, one heavy and one light, on a one-dimensional finite lattice with open boundaries, and the parameters $\alpha, \beta$, and $q$ describe the hopping probabilities. The rhombic alternative tableaux are enumerated by the Lah numbers, which also enumerate certain assemblées of permutations. We describe a bijection between the rhombic alternative tableaux and these assemblées. We also provide an insertion algorithm that gives a weight generating function for the assembées. Combined, these results give a bijective proof for the weight generating function for the rhombic alternative tableaux.

Résumé. Dans cet article, nous introduisons la notion de tableaux alternatifs rhomboïdaux, dont la série génératrice valuée permet de donner une formule combinatoire pour calculer les probabilités stationnaires du modèle ASEP avec deux types de particules. Dans ce modèle, il y a deux types de particules, lourde et légères, sur une bande finie avec extrémités ouvertes, et les paramètres $\alpha, \beta$ et $q$ décrivent les probabilités de saut. Les tableaux alternatifs romboïdaux sont énumérés par les nombres de Lah, qui aussi énumèrent les assemblées de permutations. Nous décrivons une bijection entre ces tableaux et ces assemblées. Nous aussi décrivons une algorithme d'insertion qui donne la série génératrice valuée pour les assemblées. Combinée, ces resultats donnent une preuve bijective pour la série génératrice valuée pour les tableaux alternatifs rhomboïdaux.
\end{abstract}

Keywords. rhombic alternative tableaux, Lah numbers, assemblées, ASEP, multispecies

\section{Introduction}

To motivate our introduction of the rhombic alternative tableaux, we first describe the asymmetric simple exclusion process (ASEP). The ASEP is a model from statistical physics that describes the dynamics of interacting particles hopping left and right on a one-dimensional finite lattice with open boundaries. This model was originally introduced in the 1960's by biologists and mathematicians (see for example the survey paper [3] for the connection with biology). Since its introduction, the ASEP has received a lot of attention for many reasons, including its connection to orthogonal polynomials, the XXZ model, the

\footnotetext{
†Email: olya@math.berkeley.edu

$\ddagger$ Email: viennot@xavierviennot.org
} 
formation of shocks, and more. Furthermore, it is an important example of a non-equilibrium process that exhibits boundary induced phase transitions.

The classical ASEP with three parameters is defined by the following hopping probabilities: particles may enter at the left of the lattice with rate $\alpha$, they may exit at the right with rate $\beta$, and in the bulk the probability of hopping left is $q$ times that of hopping right. The stationary probability of a state of a Markov chain is the limit as time goes to infinity of the probability of that state. In other words, the probability vector of all the states is proportional to the left eigenvector with eigenvalue 1 of the transition matrix of the Markov chain. Much past work has been devoted for finding combinatorial interpretations for the steady state probabilities of the ASEP in terms of various kinds of tableaux (permutation tableaux [7], alternative tableaux [14], tree-like tableaux [1], staircase tableaux [8]).

The most general ASEP with five parameters has a strong connection to the moments of the Askey-Wilson polynomials (see [6, 17]), which are at the top of the hierarchy of the classical orthogonal polynomials in one variable. Recently, [4, 9] revealed a fascinating connection between a two-species generalization of the ASEP with the same five parameters and moments of Koornwinder-Macdonald polynomials, an important class of multi-variate orthogonal polynomials that generalizes the Askey-Wilson polynomials. This two-species ASEP (studied in [2, 11, 16], among others), has two species of particles, heavy and light, with the heavy particles able to enter and exit at the boundaries. This recent connection with Koornwinder polynomials generated much interest in finding combinatorial objects that provide formulae for the twospecies ASEP to expand upon the results for the single-species case.

In this paper we restrict ourselves to the case of the two-species ASEP with the same three parameters $\alpha, \beta$, and $q$ as in the single-species case, as shown in Figure 1. In this model, only the heavy particles can enter or exit, so we fix the number of light particles to be $r$. When $r=0$, we reduce to the original ASEP.

In addition to the particles hopping right and left into empty locations, when the heavy and light particles are adjacent, the heavy particle treats the light particle as if it were a hole. We denote a particle by $\mathbf{\bullet}$, a light particle by $\odot$, and a hole (or absence of a particle) by $\bigcirc$. Thus the two-species ASEP is a Markov chain on the states $X \in\{\boldsymbol{O}, \bigcirc, \bigcirc\}^{n}$ with exactly $r \bigcirc$ 's. We call this set of states $B_{n}^{r}$, with $\left|B_{n}^{r}\right|=\left(\begin{array}{l}n \\ r\end{array}\right) 2^{n-r}$. See [16, 13] for the precise definitions of the transitions on $B_{n}^{r}$.

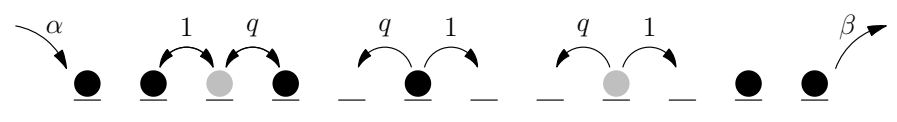

Fig. 1: The parameters of the two-species ASEP.

In [12] the first author gave an interpretation of the stationary probabilities for $q=0$ and $q=1$ with so-called multi-Catalan tableaux. Here we introduce a more natural class of tableaux, called rhombic alternative tableaux (RAT) which provide a complete combinatorial interpretation of the two-species ASEP with the three parameters $\alpha, \beta$ and $q$. These new tableaux are enumerated by the Lah numbers $\left(\begin{array}{l}n \\ r\end{array}\right)(n+1) !$. An extension of the RAT was recently used to study the combinatorics of the KoornwinderMacdonald polynomials [5].

The RAT are a two-species analog of the alternative tableaux [14] (which interpret the probabilities of the 
original ASEP). Each tableau $T$ is associated to some word $X \in\{\boldsymbol{O}, \odot, \bigcirc\}^{n}$, which we call its type. If $X$ has $r \odot$ 's, we say $T$ has size $(n, r) . T$ is a certain filling with $\alpha, \beta$, and $q$ of a closed shape with some tiling composed of rhombic cells. Each tableau has a weight $\mathrm{wt}(T)$ that is given by the product of the symbols in its filling. We define the RAT in detail in Section 2, but here we present the first main result of our paper, which is stated more precisely in Theorem 2.8

Definition 1.1 We denote by $\mathcal{Z}_{n, r}$ the weight generating function over RAT $T$ of size $(n, r)$, namely: $\mathcal{Z}_{n, r}=\sum_{X \in B_{n}^{r}} \sum_{T} \operatorname{wt}(T)$, where the second sum is over $R A T T$ of type $X$ for a fixed tiling.

Theorem 1.2 Let $X \in\{\boldsymbol{\bullet}, \bigcirc\}^{n}$ have $r \bigcirc$ 's be a state of the two-species ASEP. Then the stationary probability of state $X$ is

$$
\operatorname{Prob}(X)=\frac{1}{\mathcal{Z}_{n, r}} \sum_{T} \mathrm{wt}(T)
$$

where the sum is over RAT T of type $X$ for a fixed tiling.

The second main result of this paper is a bijection between the rhombic alternative tableaux and another combinatorial object called assemblées of permutations, which are certain collections of disjoint ordered sets. In Section 3 we describe a weighted bijection between rhombic alternative tableaux and assemblées. In Section 4 we provide an insertion algorithm for the construction of a weighted assemblée, which, combined with Section 3 , gives the following theorem.

\section{Theorem 1.3}

$$
\mathcal{Z}_{n, r}(\alpha, \beta, q=1)=(\alpha \beta)^{n-r}\left(\begin{array}{l}
n \\
r
\end{array}\right) \prod_{i=r}^{n-1}\left(\alpha^{-1}+\beta^{-1}+i\right) .
$$

For $\alpha=\beta=1$, from the above Theorem we get the Lah numbers $\left(\begin{array}{l}n \\ r\end{array}\right) \frac{(n+1) !}{(r+1) !}$.

Acknowledgement. The first author is grateful for the mentorship of Lauren Williams and Sylvie Corteel. The first author also acknowledges the hospitality of LIAFA, the Chateaubriand Fellowship, and the Berkeley-France fund, which made this work possible.

\section{Rhombic alternative tableaux}

Let $X \in\{\boldsymbol{\bullet}, \odot, \bigcirc\}^{n}$ with exactly $r \odot$ 's be a word describing a state of the two-species ASEP in $B_{n}^{r}$.

Definition 2.1 The rhombic diagram of type $X$, where $X$ has $k$ 's and $r \bigcirc$ 's with $k+r+\ell=n$, is a closed shape whose northwest boundary is a path consisting of $\ell$ west edges followed by $r$ southwest edges followed by $k$ south edges. The southeast boundary is a path that is constructed by reading $X$ from left to right and drawing a west edge for $a \bigcirc$, a southwest edge for $a \bigcirc$, and a south edge for $a$. All the edges are on a triangular lattice, and the northeast corners of the two paths are lined up (along with the southwest corners). This diagram is denoted by $\Gamma(X)$.

Definition 2.2 A tiling of $\Gamma(X)$, denoted by $\mathcal{T}$, is a covering of $\Gamma(X)$ by three types of rhombic tiles: the square, the tall rhombus, and the short rhombus, as pictured in Figure 3 The maximal tiling is one in which if tiles are placed from southeast to northwest, the tall tiles are always placed whenever possible. (In other words, the configuration of tiles on the left of Figure 5 can never occur.) 


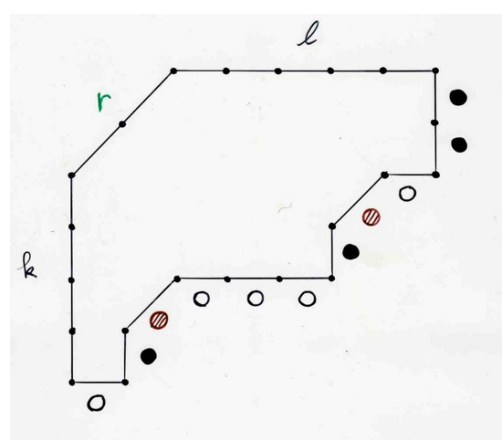

Fig. 2: $\Gamma(X)$

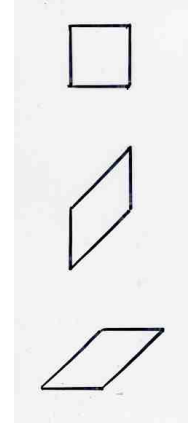

Fig. 3: Tiles

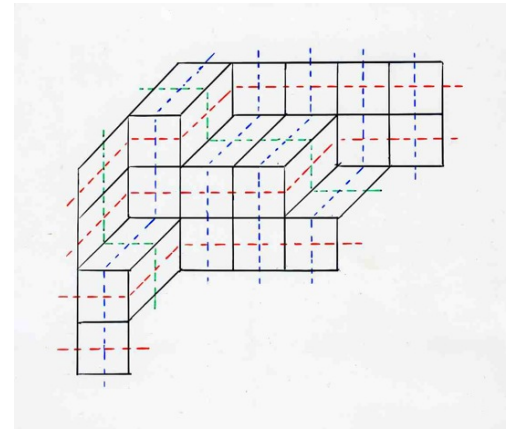

Fig. 4: West-strips (red), north-strips (blue), and northwest-strips (green).

Definition 2.3 A west-strip is a maximal connected set of tiles in which any two adjacent tiles share a vertical edge. Similarly, a north-strip is a maximal connected set of tiles in which any two adjacent tiles share a horizontal edge, and a northwest-strip is a maximal connected set of tiles in which any two adjacent tiles share a diagonal edge.

These strips can be seen in Figure 4 by following the red, blue and green lines. In a tableau of type $X$ with $k \bullet$ 's, $r \bigcirc$ 's, and $\ell \bigcirc$ 's, the total number of west-strips is $k$, the total number of north-strips is $\ell$, and the total number of northwest-strips is $r$.

Definition 2.4 Let $\mathcal{T}$ be a tiling of $\Gamma(X)$. A rhombic alternative tableau (or RAT for short) is a placement of $\alpha$ 's, $\beta$ 's, and $q$ 's in the tiles of $\mathcal{T}$ such that the following three rules are satisfied:

$i$. Any tile left of and in the same west-strip as a $\beta$ is empty.

$i$. Any tile above and in the same north-strip as an $\alpha$ is empty.

iii. There are no other empty tiles.

We see an example of such a tableau in Figure 6 We denote by $R(\mathcal{T})$ the set of RAT associated to the tiling $\mathcal{T}$ of the diagram $\Gamma(X)$.

Definition 2.5 Let $T$ be a RAT of type $X$, where $X$ has $k$ 's, $r \bigcirc$ 's, and $\ell \bigcirc$ 's. Then the weight of $T$ is defined as:

$$
\operatorname{wt}(T)=\alpha^{k} \beta^{\ell} \cdot(\text { product of the symbols within } T)
$$

Example. For the tableau in Figure 6, we have $\operatorname{wt}(T)=\alpha^{4} \beta^{4} \cdot \alpha^{3} \beta^{2} q^{15}$.

Definition 2.6 We define weight ${ }_{\mathcal{T}}(X)$ to be the weight generating function of all RAT with tiling $\mathcal{T}$, namely

$$
\text { weight }_{\mathcal{T}}(X)=\sum_{T \in R(\mathcal{T})} \mathrm{wt}(T),
$$

Lemma 2.7 For two tilings $\mathcal{T}$ and $\mathcal{T}^{\prime}$,

$$
\text { weight }_{\mathcal{T}}(X)=\text { weight }_{\mathcal{T}^{\prime}}(X) .
$$


The lemma is proved by first showing that any tiling can be obtained from another via a series of "flips" (see Figure 5), and then a weight-preserving involution is constructed between fillings of tilings that differ by a single flip. There are 12 different possible configurations of a minimal hexagon with filling of $\alpha, \beta$, and $q$ 's in the tiles. For each of them we define an involution $\phi$ associating a maximal hexagon with a certain filling of $\alpha, \beta$, and $q$ 's. Figure 5 shows two of these 12 possible cases.

By Lemma 2.7, we are able to define weight $(X)=$ weight $_{\mathcal{T}}(X)$ for an arbitrary $\mathcal{T}$. Our main result for this section is the following.

Theorem 2.8 Let $X \in\{\bullet, \bigcirc, \bigcirc\}^{n}$ with exactly $r \bigcirc$ 's be a word representing a state of the two-species ASEP of size $(n, r)$. Define $\mathcal{Z}_{n, r}=\sum_{X \in B_{n}^{r}}$ weight $(X)$. Then the steady state probability of state $X$ is

$$
\operatorname{Prob}(X)=\frac{1}{\mathcal{Z}_{n, r}} \operatorname{weight}(X)
$$

A complete proof of this theorem in terms of a Matrix Ansatz [16] can be found in the preprint [13], which is the full version of Sections 1 and 2 of this extended summary. It would be possible to give another proof of Theorem 2.8 in the spirit of the general theory called cellular ansatz, introduced and developed by the second author in [15]. The Matrix Ansatz for the two-species ASEP defines an algebra with three generators $D, E$, and $A$ and three commutation relations $D E=q E D+E+D, D A=q A D+A$, and $A E=q E A+A$. This quadratic algebra does not quite fit into the general cellular ansatz theory of Chapter 6a of [15], but the theory could be extended to such an algebra by replacing the quadratic lattice by a tiling $\mathcal{T}$ of the diagram $\Gamma(X)$. The corresponding $Q$-tableaux (so-called from the general theory of the cellular ansatz) are the RAT, and in a similar way, one can prove that any word $\mathrm{W}$ in letters $\{D, A, E\}$ can be expressed in a unique way as a sum of monomials $q^{t} D^{i} A^{r} D^{j}$ over $T \in R(\mathcal{T})$ for $\mathcal{T}$ a tiling of $\Gamma(W)$. (Here $i$ (respectively $j$ ) is the number of north-strips (respectively west-strips) of $T$ not containing an $\alpha$ (respectively $\beta$ ), and $t$ is the number of cells weighted $q$.)

\section{Bijection from rhombic alternative tableaux to assemblées}

The alternative tableaux (corresponding to rhombic alternative tableaux with $r=0$ ) are enumerated by $n$ !, and are in bijection with permutations. In [14], the second author provided a weight-preserving bijection from permutations of $n+1$ to alternative tableaux of size $n$. This bijection is called exchange-fusion.

The rhombic alternative tableaux are enumerated by the Lah numbers, which are indexed by $n, k$ and defined as $\left(\begin{array}{l}n \\ r\end{array}\right) \frac{(n+1) !}{(r+1) !}$. The Lah numbers also enumerate assemblées of permutations, which are certain collections of permutations. We will refer to them as simply assemblées. In this section, we will describe a weight preserving bijection between assemblées and the rhombic alternative tableaux, that generalizes the exchange-fusion algorithm of [14] for the alternative tableaux.

Definition 3.1 An assemblée of size $(n, r)$ is a collection of $r$ nonempty ordered sets, or blocks, consisting of elements in $\{1, \ldots, n\}$, where the sets are all disjoint, and their union is $\{1, \ldots, n\}$. We call the last element in each block the block-end, and we give a canonical order to the blocks such that the block-ends are decreasing from left to right. 
Example. $A=[2,10,12,7][5,9,1,8,6][3,11,4]$ is an example of an assemblée of size $(12,3)$ in canonical order, and the block-ends are $[7,6,4]$.

Definition 3.2 Let $A$ be an assemblée of size $(n, r)$. We define the block-end sequence $\mathbf{b}=\left[b_{1}, \ldots, b_{r}\right]$ to be the decreasing sequence of block-ends of $A$. Now considering the permutation obtained by deleting the parentheses in $A$, written in canonical order, we define the left-right sequence to be the sequence of left to right maximal elements greater than $b_{1}$, and we call it $\operatorname{rls}(A)$. Similarly, we define the right-left sequence to be the sequence of right to left maximal elements smaller than $b_{r}$, and we call it $\operatorname{lrs}(A)$.

Example. For $A=[2,10,12,7][5,9,1,8,6][3,11,4]$, we have $\operatorname{rls}(A)=[12,11]$ and $\operatorname{lrs}(A)=[3,2]$.

The goal of this section is to describe a bijection between rhombic alternative tableaux with weight $\alpha^{n-r-i} \beta^{n-r-j}$ at $q=1$ and assemblées with $|\operatorname{rls}|=i$ and $|\operatorname{lrs}|=j$.

\subsection{Assemblées to RAT with the exchange-fusion rewriting algorithm}

Let $A$ be an assemblée of size $(n+1, r+1)$ with block-end sequence $\mathbf{b}$. We also make the following definitions:

Definition 3.3 Suppose $x \in A$ is not a block-end. If $x+1$ is to the right of $x$ in $A$, then $x$ is an increase. Otherwise, $x$ is a decrease. By convention, $n+1$ is a decrease if it is not a block-end.

Example. For $A=[2,10,12,7][5,9,1,8,6][3,11,4]$, the set of increases is $\{2,10,5,3\}$, and the set of decreases is $\{12,9,1,8,11\}$.

We associate to an assemblée $A$ (in the canonical order) of size $(n+1, r+1)$ a state $X(A) \in B_{n}^{r}$ of the two-species ASEP by replacing each decrease with a $\bigcirc$, each increase with a $\bullet$, and each block-end with a $\bigcirc$, with the last block-end omitted.

Example. For $A=[2,10,12,7][5,9,1,8,6][3,11,4]$, we have $X(A)=\bullet \bullet \bigcirc \bullet \bigcirc \bigcirc \bigcirc \bigcirc \bigcirc$.

Let $A$ be an assemblée of size $(n+1, r+1)$. We describe an exchange-fusion algorithm to build a RAT $T(A)$ of type $X(A)$ and size $(n, r)$. In this algorithm, we are filling a rhombic diagram of type $X(A)$ with an arbitrary tiling, and then send labels through the edges of the tiles from the southeast boundary to the northwest boundary of $\Gamma(X(A))$.

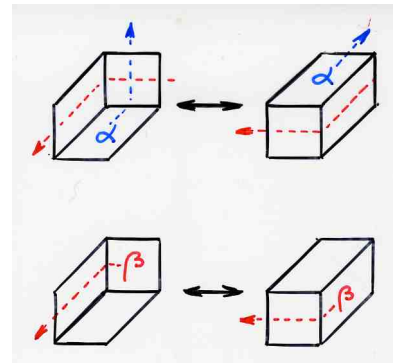

Fig. 5: Two cases of flips for the involution $\phi$

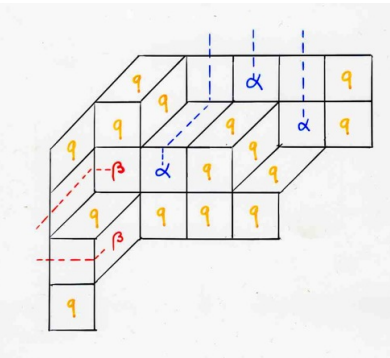

Fig. 6: A rhombic alternative tableau 
Definition 3.4 $A$ label is a (possibly empty) set of consecutive integers. For two disjoint nonempty labels $A$ and $B$, we say $B \succ A$ if for the smallest $i \in B$ and the largest $j \in A, i=j+1$.

Note that if $B \succ A$, then $A \cup B$ is also a label. Moreover, by convention, for any label $A, A \nsucc \emptyset$.

Initiation. We set the label of every edge on the southeast boundary of $\Gamma(X(A))$ to be the corresponding element of $A$. For example, see Figure 7

Step. Let a tile have its east-most edge and south-most edge have labels $A$ and $B$. Then we label the west-most edge and north-most edge according to the following cases.

i. $A \nsucc B$ and $B \nsucc A$. Then the labels simply pass through each other, and the west-most edge receives the label $A$ and the north-most edge receives the label $B$. A $q$ is placed in the tile.

ii. $A \succ B$ and $B$ is labeling a horizontal edge. Then the west-most edge receives the label $B \cup A$, the north-most edge receives the label $\emptyset$, and an $\alpha$ is placed in the tile.

iii. $B \succ A$ and $A$ is labeling a vertical edge. Then the north-most edge receives the label $A \cup B$, the west-most edge receives the label $\emptyset$, and a $\beta$ is placed in the tile.

iv. $A \succ B$ and $B$ is labeling a diagonal edge. Then the labels simply pass through each other, and the west-most edge receives the label $A$ and the north-most edge receives the label $B$.

An example of this algorithm can be seen in Figure 7. At the end of the process we get a RAT $T(A)$ of type $X(A)$ and size $(n, r)$. Furthermore, one can prove that if $|\operatorname{rls}(A)|=i$ and $|\operatorname{lrs}(A)|=j$, then $\operatorname{wt}(T(A))=\alpha^{n-r-i} \beta^{n-r-j}$ at $q=1$.

\subsection{Rhombic alternative tableaux to assemblées}

In this section, we give an algorithm that accomplishes the reverse of Section 3.1. Let $T$ be a RAT of type $X$ with arbitrary tiling $\mathcal{T}$ and size $(n, r)$, such that $X$ has $k$ 's and $\ell \bigcirc$ 's (with $k+\ell+r=n$ ). We will construct from $T$ an assemblée $A(T)$ of size $(n+1, r+1)$.

First we associate to $T$ a forest of crossing binary trees, starting with a network of lines passing through the west-, north- and northwest- strips as in Figure 4 . For each west-strip, we draw a line through the midpoints of the vertical edges of each tile of the strip, indicated by red in Figure 8 We draw corresponding lines for the north-strips and the north-west strips, indicated by blue and green respectively in Figure 8 .

For each tile labeled $\alpha$, we remove the section of the blue line north of that $\alpha$. Similarly, for each tile labeled $\beta$, we remove the section of the red line west of that $\beta$. We obtain a forest of binary trees, where the vertices (or branching nodes) of the trees are the tiles labeled $\alpha$ and $\beta$. Moreover, a tile contains two intersecting branches (either from different trees or from the same tree) if and only if it is labeled with a $q$. Finally, each tree has a root edge starting on the northwest border of the diagram $\Gamma(X)$ (see Figure 8 . The root of each tree is either a red, blue, or green line (coming from a west-, north-, or northwest- strip, respectively). Thus we say the trees belong to three classes red, blue, or green. By our convention, we also add a special trivial green tree to the southwest point of our tableau. In Figure 8 , this trivial tree is denoted by the label "(4)". Note that for a RAT of size $(n, r)$ with $i$ north-strips with no cell labeled $\alpha$ and $j$ west-strips with no cell labeled $\beta$, there will be $i$ trees with a blue root, $j$ trees with a red root, and $r+1$ trees with a green root (including the special trivial tree). 
Remark 3.5 The binary trees are drawn on the tiling $\mathcal{T}$, and a flip on a tiling will correspond to a certain local move on the branches of the tree similar to a Yang-Baxter move.

The roots of the three classes of binary trees, including the special trivial green tree, are totally ordered. In this ordering, we consider first the $j$ red roots from top to bottom, then the $r+1$ green roots from southwest to northeast, and finally the $i$ blue roots from left to right. We label each root edge of a binary tree $\mathcal{B}$ by some label $B$ according to the three following conditions:

- The size of $B$ is one if it is the special trivial tree, otherwise it equals the number of external vertices of $\mathcal{B}$ (that is the number of edges on southeast boundary that are connected to the root edge of $\mathcal{B}$ via that binary tree).

- For any two consecutive root edges $R$ and $R^{\prime}$ (where $R^{\prime}$ follows $R$ in the total order) the corresponding labels $B$ and $B^{\prime}$ satisfy $B^{\prime} \succ B$.

- The union of the labels of the root edges is $\{1, \ldots, n+1\}$.

We see an example of the labeling of the root edges in Figure 8

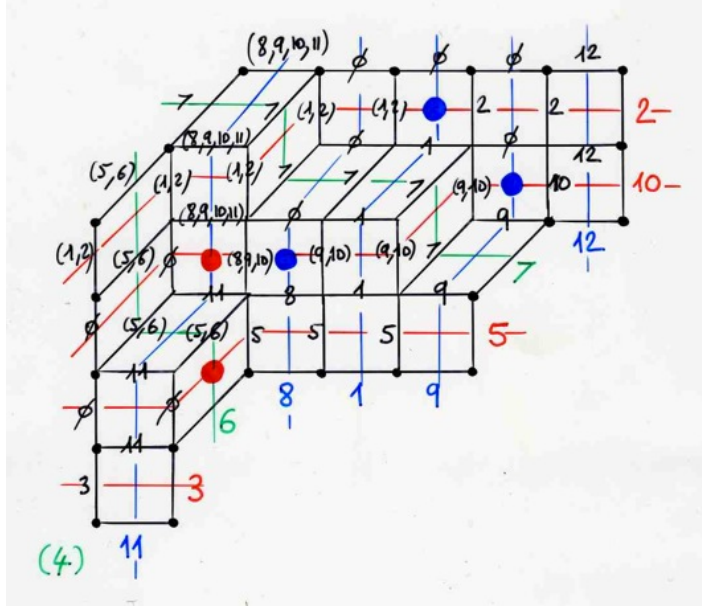

Fig. 7: The exchange-fusion algorithm

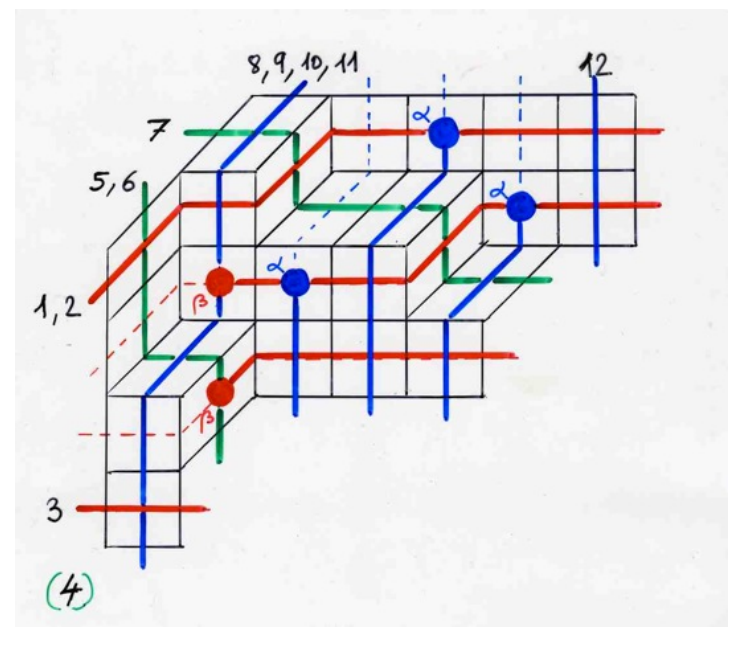

Fig. 8: Forest of binary trees

The labels are sent along the branches to the southeast. Whenever a label $B$ is at a vertex, it is split into two disjoint labels $C$ and $D$ such that $C \cup D=B$. Moreover, the branch that receives the label $C$ must have $|C|$ edges on the southeast boundary connected to it, and the branch that receives the label $D$ must consequently have $|D|$ edges on the southeast boundary connected to it. There is one final rule:

- if the vertex is located within a northwest-strip, then the branch that is going in the southeast direction out of that vertex receives the larger label.

- otherwise, if the vertex corresponds to an $\alpha$, then the branch going in the east direction out of the vertex receives the larger label, and the branch going in the south direction out of the vertex receives the smaller label. 
- similarly, if the vertex corresponds to an $\beta$, then the branch going in the south direction out of the vertex receives the larger label, and the branch going in the east direction out of the vertex receives the smaller label.

The algorithm is complete once every edge on the southeast boundary has received a label that consists of a single integer. An assemblée is then obtained by reading the edge labels from northeast to southwest, and assigning the labels of the diagonal edges to be the block-ends. The final block-end is assigned to be the integer in $\{1, \ldots, n+1\}$ that does not appear in the edge labels of the tableau, that is the label of the special trivial root added at the beginning.

One can prove that the two above algorithms between rhombic alternative tableaux of size $(n, r)$ and assemblées of size $(n+1, r+1)$ are bijections that are also inverses of each other. Furthermore, for the RAT $T$ and its corresponding assemblée $A=A(T)$, one can prove that the number $i$ of blue root vertices is $|\operatorname{rls}(A)|$, and the number $j$ of red root vertices is $|\operatorname{lrs}(A)|$. More specifically, the sequence of maximal elements in the label of each blue root is precisely $\operatorname{rls}(A)$, and the sequence of maximal elements in the label of each red root is precisely $\operatorname{lrs}(A)$ in $A$. Thus we obtain the following proposition.

Proposition 3.6 There is a bijection between RAT in $B_{n}^{r}$ and assemblées in $\mathcal{A}_{n+1, r+1}$. Moreover, in this bijection, $T \in B_{n}^{r}$ with weight $\alpha^{n-r-i} \beta^{n-r-j}$ at $q=1$ corresponds to $A \in \mathcal{A}_{n+1, r+1}$ with $|\operatorname{rls}|=i$ and $|\operatorname{lrs}|=j$.

\section{Weighted enumeration of assemblées}

In this section, we provide a weight-preserving bijection between assemblées and a pair composed by the choice of a subset $\left(\begin{array}{l}n \\ r\end{array}\right)$ and an $r$-truncated subexceedant function on $[n-r]$, which is enumerated by the product $(r+2) \ldots(n+1)$. This leads to a bijective proof of the following Theorem.

Theorem 4.1 Let $A \in \mathcal{A}_{n+1, r+1}$ be an assemblée. Then

$$
\sum_{A: \operatorname{size}(A)=(n+1, r+1)} \alpha^{-\operatorname{rls}(A)} \beta^{-\operatorname{lrs}(A)}=\left(\begin{array}{l}
n \\
r
\end{array}\right) \prod_{i=r}^{n-1}\left(\alpha^{-1}+\beta^{-1}+i\right) .
$$

Definition 4.2 A subexceedant function on $[n]=[1, \ldots, n]$ is a function $f:[n] \rightarrow[n]$ such that $f(i) \leq i$ for each $i \in[n]$. An $r$-truncated subexceedant function is such a function $f$ with $f(i) \leq i+r+1$.

We define an insertion algorithm as follows. Let $f$ be an $r$-truncated subexceedant function on $[n-r]$. We begin with $r+1$ horizontal green lines at heights 1 through $r+1$ from bottom to top. For $i \in[n-r]$, we insert element $i$ to the right of element $i-1$ in position $f(i)$ relative to the $r+1$ green lines and the elements $[i-1]$ which have already been inserted. In other words, if $f(i)=1$, then $i$ is inserted below the other elements and the green lines, and if $f(i)=k$, then $i$ is inserted above the element at height $k-1$.

Once all elements have been inserted, each is assigned a value from $[n+1]$ that corresponds to its height relative to the other elements and the green lines. Finally, a point is chosen on each of the green lines such that the points are located from top to bottom when read from left to right, and the point on the bottom-most green line is fixed to be to the right of $n-r$. This selection fixes the locations of the blockends in the assemblée. Figure 9 shows an example of this insertion of size $(11,3)$, where the 3 -truncated 
subexceedant function $f$ is defined by $f(1)=3, f(2)=5, f(3)=2, f(4)=6, f(5)=1, f(6)=$ $9, f(7)=2, f(8)=1$.

It is easy to check that an $r$-truncated subexceedant function $f$ combined with a choice of the positions of the points on the green lines results in an assemblée $A \in \mathcal{A}_{n+1, r+1}$. Note that there are $\left(\begin{array}{l}n \\ r\end{array}\right)$ ways to choose the points on the green lines, and each of those choices results in a distinct assemblée.

For the $r$-truncated subexceedent function $f$, the inserted elements are given colors and weights as follows:

- When $f(i)=1$, the $i$ 'th inserted element is colored red and given weight $\beta^{-1}$.

- When $f(i)=i+r+1$, the $i$ 'th inserted element is colored blue and given weight $\alpha^{-1}$.

- Otherwise, the element is colored black and given weight 1.

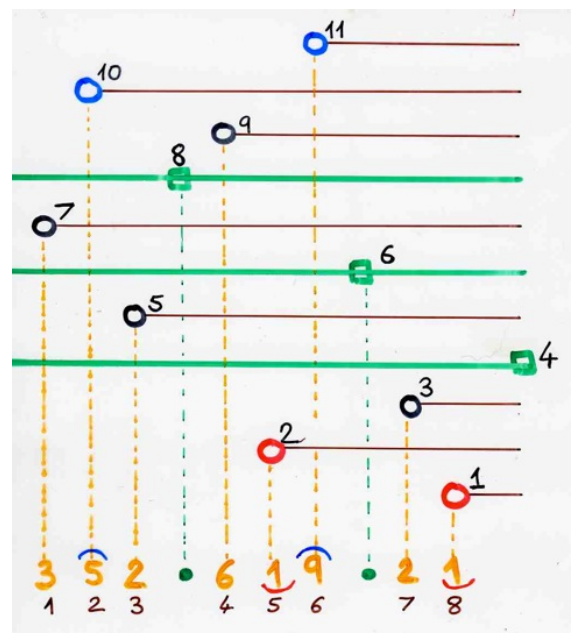

Fig. 9: Insertion of 8 elements to obtain the assemblée $[7,10,5,8][9,2,11,6][3,1,4]$.

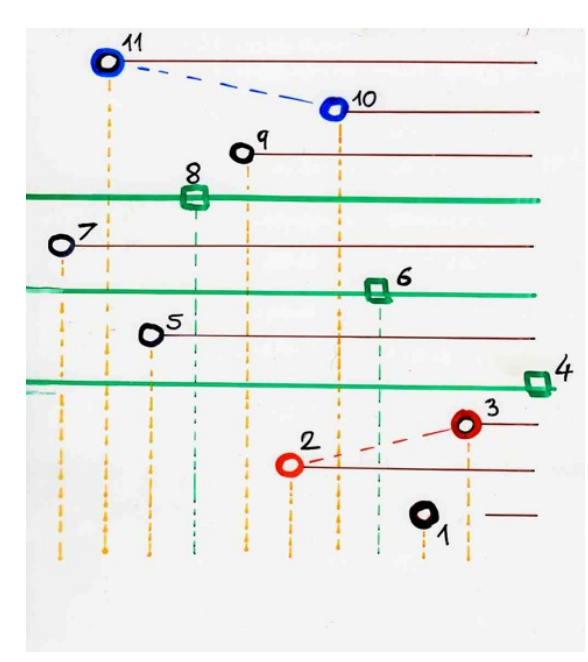

Fig. 10: The transformation $\rho$.

Definition 4.3 The total weight of the final list is the product of the weights of all the inserted elements.

For example, the weight of the assemblée constructed in Figure 9 is $\alpha^{-2} \beta^{-2}$.

Let $b_{i}$ be the relative height of the $i$ 'th green line from top to bottom for $1 \leq i \leq r+1$, and let $x_{i}$ be the relative height of the $i$ 'th inserted element for $1 \leq i \leq n-r$. An assemblée $A$ is obtained by merging the sequence $\left[x_{1}, \ldots, x_{n-r}\right]$ with the sequence $\left[b_{1}, \ldots, b_{r+1}\right]$ so that the values $b_{i}$ are in the locations corresponding to the relative lateral placement of the green points in between the $x_{i}$ 's, and they are set to be the block-ends of $A$ (See Figure 9). Now, this $A$ is almost an assemblee of size $(n+1, r+1)$ with the desired weight. To obtain the correct weight, we apply the transformation $\rho$ to $A$ guarantee that:

- The number of times an inserted element $x \in A$ was given weight $\alpha^{-1}$ equals $\operatorname{lrs}(\rho(A))$.

- The number of times an inserted element $x \in A$ was given weight $\beta^{-1}$ equals $\operatorname{rls}(\rho(A))$. 
Definition 4.4 Let $\mathbf{b}=\left[b_{1}, \ldots, b_{r+1}\right]$ be the sequence of block-end elements of $A$. Let the sequence $\operatorname{large}(A)=\left[a_{1}, a_{2}, \ldots, a_{u}\right]$ consist of all the elements $a_{i} \in A$ such that $a_{i}>b_{1}$, where the order of the $a_{i}$ 's matches the order of their appearance in A from left to right. Similarly, let the sequence $\operatorname{small}(A)=\left[c_{1}, c_{2}, \ldots, c_{w}\right]$ consist of all the elements $c_{i}$ such that $c_{i}<b_{r+1}$, where the order of the $c_{i}$ 's matches the order of their appearance in A from left to right. We define $\rho(A)$ as the transformation that replaces $a_{i}$ with $a_{u-i+1}$ for $1 \leq i \leq u$, replaces $c_{i}$ with $b_{r+1}-c_{i}$ for $1 \leq i \leq w$, and leaves the rest of the entries of $A$ unchanged.

Remark 4.5 On large $(A)$, the transformation $\rho$ acts as the classical operation "mirror image" (in Figure 10. this means the points in large $(A)$ are reflected across a vertical axis), and on small $(A), \rho$ acts as the classical operation "complement" (in Figure 10 this means the points in $\operatorname{small}(A)$ are reflected across a horizontal axis).

Example. The definition of $\rho(A)$ is best illustrated by an example. For $A=[7,10,5,8][9,2,11,6][3,1,4]$ with $\mathbf{b}=[8,6,4]$ of Figure 9, we have $\operatorname{large}(A)=[10,9,11]$ and $\operatorname{small}(A)=[2,3,1]$. In Figure 10, we obtain $\rho(\operatorname{large}(A))=[11,9,10]$ and $\rho(\operatorname{small}(A))=[2,1,3]$. Thus we obtain as desired $\rho(A)=[7,11,5,8][9,2,10,6][1,3,4]$ with $\operatorname{lrs}(\rho(A))=[10,11]$ and $\operatorname{rls}(\rho(A))=[2,3]$.

Theorem 4.6 Let $A$ be an assemblée resulting from applying the insertion algorithm, and let $\mathrm{wt}_{\beta}$ and $\mathrm{wt}_{\alpha}$ be the number of times a non-block-end element $x \in A$ was given weight $\beta^{-1}$ and $\alpha^{-1}$ respectively. Then $\operatorname{lrs}(\rho(A))=\mathrm{wt}_{\alpha}$ and $\operatorname{rls}(\rho(A))=\mathrm{wt}_{\beta}$.

\section{Conclusion and further results}

Recall that in Section 1 , we defined $\mathcal{Z}_{n, r}$ to be the sum over all tableaux $T$, where each $T$ has a type that is a state of the two-species ASEP $X \in B_{n}^{r}$, and such that for each $X$ a tiling $\mathcal{T}$ is fixed for $\Gamma(X)$. $\mathcal{Z}_{n, r}$ is also the partition function of the two-species ASEP with three parameters $\alpha, \beta$ and $q$ (i.e. the sum over the unnormalized steady state probabilities of all the states in $B_{n}^{r}$ ).

By combining the three bijections presented in Sections 3 and 4 of this paper, we get a bijective proof of the formula for the weight generating function for RAT with parameters $\alpha$ and $\beta$ :

$$
\mathcal{Z}_{n, r}(\alpha, \beta, q=1)=(\alpha \beta)^{n-r}\left(\begin{array}{l}
n \\
r
\end{array}\right) \prod_{i=r}^{n-1}\left(\alpha^{-1}+\beta^{-1}+i\right)
$$

which is also the partition function of the two-species ASEP at $q=1$.

It remains to find an interpretation of the parameter $q$ in terms of assemblées. Furthermore, from the forest of binary trees of Section 3.2, we can define an analog of the tree-like tableaux of [1]. This tree-like analog on the RAT has vertices in each cell of the RAT $T$ containing an $\alpha$ or $\beta$, and also at every edge on the north-west boundary of $T$ that is associated to a root edge, as in Figure 8 . Such tableaux are in bijection with the RAT. From this paper, an analog of the insertion algorithm of [1] should be defined for rhombic tree-like tableaux which would lead to a combinatorial interpretation of the parameter $q$ in terms of assemblées of permutations. 


\section{References}

[1] J. Aval, A. Boussicault, P. Nadeau. Tree-like tableaux. Electr. J. Combin. 20-4, 4, P. 34, 24, (2013).

[2] C. Arita, A. Ayyer, J. L. Leibowitz, E. R. Speer. On the two species asymmetric exclusion process with semi-permeable boundaries. J. Stat. Phys. 135, no. 5-6, 1009-1037 (2009).

[3] A. Blythe and M. R. Evans, Nonequilibrium steady states of matrix-product form: a solver's guide, Journal of Physics A: Mathematical and Theoretical: 40, no. 46, R333, (2007).

[4] L. Cantini, Asymmetric simple exclusion process with open boundaries and Koornwinder polynomials, arXiv: 1506.00284 [math.CO], (2015).

[5] S. Corteel, O. Mandelshtam, and L. Williams. Combinatorics of the two-species ASEP and Koornwinder polynomials, arXiv: 1510.05023 [math.CO], (2015).

[6] S. Corteel, R. Stanley, D. Stanton, L. Williams. Formulae for Askey-Wilson moments and enumeration of staircase tableaux. Trans. Amer. Math. Soc., 364(11):6009-6037, (2012).

[7] S. Corteel and L. Williams. Tableaux combinatorics for the asymmetric exclusion process, Advances in Applied Mathematics, Volume 39, Issue 3, 293-310, (2007).

[8] S. Corteel and L. Williams. Tableaux combinatorics for the asymmetric exclusion process and Askey-Wilson polynomials. Duke Math. J., 159: 385-415, (2011).

[9] S. Corteel and L. Williams, Koornwinder moments and the two-species exclusion process, arXiv: 1505.00843 [math.CO], (2015).

[10] B. Derrida, M. Evans, V. Hakim, V. Pasquier, Exact solution of a 1D asymmetric exclusion model using a matrix formulation, J. Phys. A: Math. Gen. 26, 1493-1517 (1993).

[11] E. Duchi, G. Schaeffer, A combinatorial approach to jumping particles, J. Combin. Theory Ser. A 110, no.1, 1-29, (2005).

[12] O. Mandelshtam, Multi-Catalan Tableaux and the Two-Species TASEP, extended abstract in DMTCS Proc. FPSAC'15, 685-696, (2015).

[13] O. Mandelshtam and X. Viennot. Tableaux combinatorics for the two-species PASEP, to appear in J. Combin. Theory Ser. A, (2015).

[14] X. Viennot, Alternative tableaux, permutations and partially asymmetric exclusion process. Workshop at "Stat. Mech. and Quantum-Field Theory Methods in Comb. Enumeration", Isaac Newton Inst. for Mat. Sci., Cambridge, Apr. 2008, slides at: http://sms . cam.ac.uk/media/1004.

[15] X. Viennot, Algebraic combinatorics and interactions: the cellular ansatz. Course at IIT Bombay, Jan. 2013, slides at: http:// cours.xavierviennot.org/IIT_Bombay_2013.html.

[16] M. Uchiyama. Two-Species Asymmetric Simple Exclusion Process with Open Boundaries. Chaos, Solitons and Fractals, 35, no. 2, 398-407 (2008).

[17] M. Uchiyama, T. Sasamoto, and M. Wadati. Asymmetric simple exclusion process with open boundaries and Askey-Wilson polynomials. J. Phys. A, 37(18):4985-5002, (2004). 